\title{
Pair Excitations and Vertex Corrections in Fermi Fluids
}

\author{
Helga M. Böhm, Eckhard Krotscheck, and Martin Panholzer \\ Inst. f. Theoretische Physik, Johannes Kepler Universität, A-4040 Linz, Austria
}

Based on an equations-of-motion approach for time-dependent pair correlations in strongly interacting Fermi liquids, we have developed a theory for describing the excitation spectrum of these systems. Compared to the known "correlated" random-phase approximation (CRPA), our approach has the following properties: i) The CRPA is reproduced when pair fluctuations are neglected. ii) The first two energy-weighted sumrules are fulfilled implying a correct static structure. iii) No ad-hoc assumptions for the effective mass are needed to reproduce the experimental dispersion of the roton in ${ }^{3} \mathrm{He}$. iv) The density response function displays a novel form, arising from vertex corrections in the proper polarisation. Our theory is presented here with special emphasis on this latter point. We have also extended the approach to the single particle self-energy and included pair fluctuations in the same way. The theory provides a diagrammatic superset of the familiar $G W$ approximation. It aims at a consistent calculation of single particle excitations with an accuracy that has previously only been achieved for impurities in Bose liquids.

PACS numbers: 67.55.-s, 67.55.Jd, 671.10Ca

\section{MOTIVATION}

Whereas the ground state properties of strongly interacting fermionic fluids are nowadays well understood, the features of higher-lying excitations such as the roton in He and the plasmon in electron liquids still require further clarification. Without a proper consideration of pair excitations, both the dispersion and the damping of these collective modes are described 


\section{H. M. Böhm, E. Krotscheck, and M. Panholzer}

incorrectly. In the bosonic case, in particular for the roton in ${ }^{4} \mathrm{He}$, the introduction of "backflow" correlations (1] (equivalent to a special case of pair excitations) has led to a theory ${ }^{233}$ yielding remarkable agreement with experiment. The correction of the roton position is due to pair fluctuations which have wavelengths comparable to the interparticle distance.

In Fermi liquids the density-density response function is conventionally taken to be of the CRPA form

$$
\chi^{\mathrm{CRPA}}(q, \omega)=\chi_{0}(q, \omega) /\left(1-\widetilde{V}_{\mathrm{p}-\mathrm{h}}(q) \chi_{0}(q, \omega)\right),
$$

where $\chi_{0}$ is the Lindhard function and $\widetilde{V}_{\mathrm{p}-\mathrm{h}}$ a suitable effective interaction (also termed ,,pseudopotential ${ }^{\sqrt[5]{ }}$ " and ,local field ${ }^{6}$ corrected potential”). Fixing $\widetilde{V}_{\mathrm{p}-\mathrm{h}}$ through the $\omega^{0}$ and $\omega^{1}$ sum rules leads to a collective mode that is energetically much higher than found in experiments. One can cure this problem in an $a d$-hoc manner by introducing an average effective mass ${ }^{*}{ }^{*}$ in $\chi_{0}$. This is unsatisfactory from the point of view of developing a manifestly microscopic description of the low-temperature properties of ${ }^{3} \mathrm{He}$, and it also introduces inconsistencies since it violates the above sum rules. In addition, one would expect that the physical mechanisms that lead to the lowering of the phonon-roton spectrum in ${ }^{4} \mathrm{He}$ from the Feynman dispersion law are also at work in ${ }^{3} \mathrm{He}$. As in ${ }^{4} \mathrm{He}$ the phonon-roton spectrum has been understood quantitatively without the violation of the two above sumrules, our goal is here to develop a theory for ${ }^{3} \mathrm{He}$ that has the same level of accuracy and consistenty as the ${ }^{4}$ He theory.

The need for such a theory is made even clearer in quasi-2-dimensional ${ }^{3} \mathrm{He}$, where the collective mode is found inside the particle-hole continuum ${ }^{7}$. An effective $m^{*}$ in an RPA formula (11) may lower (both!) the continuum and the collective mode, but only dynamic correlations could reproduce the broadening of the phonon.

\section{FORMALISM}

We describe the excited state wave function of strongly interacting fermions by a generalized time-dependent Hartree-Fock form

$$
|\Psi(t)\rangle=\frac{1}{\mathcal{N}^{1 / 2}} e^{-i H_{00} t / \hbar} F e^{U(t)}\left|\Phi_{0}\right\rangle,
$$

where $\mathcal{N}$ denotes the normalization integral, $\left|\Phi_{0}\right\rangle$ the Slater determinant and $H_{00}$ the energy of the correlated ${ }^{8}$ ground state $F\left|\Phi_{0}\right\rangle$. The excitation operator $U$ includes both single-pair ("c") and two-pair (" $d$ ") amplitudes:

$$
U(t)=\sum_{p h} c_{p h}(t) a_{p}^{\dagger} a_{h}+\frac{1}{2} \sum_{p h h^{\prime} p^{\prime}} d_{p p^{\prime} h h^{\prime}}(t) a_{p}^{\dagger} a_{p^{\prime}}^{\dagger} a_{h^{\prime}} a_{h},
$$




\section{Pair Excitations in Fermi Fluids}

The amplitudes $c_{p h}(t)$ and $d_{p p^{\prime} h h^{\prime}}(t)$ are determined by minimizing the action integral corresponding to the time-dependent Schrödinger equation. Treating the external field $h^{\text {ext }}$ and consequently $c_{p h}$ and $d_{p p^{\prime} h h^{\prime}}$ as first order perturbations, the resulting equations of motion (EOMs) read

$$
\begin{aligned}
& {\left[i \hbar \frac{\partial}{\partial t}-e_{p h}\right] c_{p h}(t)=h_{p h}^{\mathrm{ext}}(t)+\left\langle p h^{\prime}\left|\widetilde{V}_{\mathrm{p}-\mathrm{h}}\right| h p^{\prime}\right\rangle_{a} c_{p^{\prime} h^{\prime}}+} \\
& \left\langle p p^{\prime}\left|\widetilde{V}_{\mathrm{p}-\mathrm{h}}\right| h h^{\prime}\right\rangle_{a} c_{p^{\prime} h^{\prime}}^{*}+\left\langle p h^{\prime}|W| p^{\prime \prime} p^{\prime}\right\rangle_{a} d_{p^{\prime \prime} p^{\prime}, h h^{\prime}}-\left\langle h^{\prime \prime} h^{\prime}|W| h p^{\prime}\right\rangle_{a} d_{p p^{\prime}, h^{\prime \prime} h^{\prime}},
\end{aligned}
$$

with $e_{p h} \equiv e_{p}-e_{h}$ denoting the particle-hole excitation energies and $W$ the effective interaction in the channels other than particle-hole $\left(\widetilde{V}_{\mathrm{p}-\mathrm{h}}\right)$, particleparticle $\left(\widetilde{V}_{\mathrm{p}-\mathrm{p}}\right)$, and hole-hole $\left(\widetilde{V}_{\mathrm{h}-\mathrm{h}}\right)$;

$$
\begin{aligned}
& {\left[i \hbar \frac{\partial}{\partial t}-e_{p h}-e_{p^{\prime} h^{\prime}}\right] d_{p p^{\prime}, h h^{\prime}}=} \\
& \left\langle p p^{\prime}|W| h p^{\prime \prime}\right\rangle c_{p^{\prime \prime} h^{\prime}}+\left\langle p p^{\prime}|W| p^{\prime \prime} h^{\prime}\right\rangle c_{p^{\prime \prime} h}-\left\langle p h^{\prime \prime}|W| h h^{\prime}\right\rangle c_{p^{\prime} h^{\prime \prime}}-\left\langle h^{\prime \prime} p^{\prime}|W| h h^{\prime}\right\rangle c_{p h^{\prime \prime}} \\
& \quad+\left[\left\langle h^{\prime \prime} p^{\prime}\left|\widetilde{V}_{\mathrm{p}-\mathrm{h}}\right| p^{\prime \prime} h^{\prime}\right\rangle_{a} d_{p p^{\prime \prime}, h h^{\prime \prime}}-\left\langle h^{\prime \prime} p^{\prime}\left|\widetilde{V}_{\mathrm{p}-\mathrm{h}}\right| h p^{\prime \prime}\right\rangle_{a} d_{p p^{\prime \prime}, h^{\prime \prime} h^{\prime}}+\left(\begin{array}{c}
p \leftrightarrow p^{\prime} \\
h \leftrightarrow h^{\prime}
\end{array}\right)\right] \\
& \quad+\left\langle p p^{\prime}\left|\widetilde{V}_{\mathrm{p}-\mathrm{p}}\right| p^{\prime \prime} p^{\prime \prime \prime}\right\rangle d_{p^{\prime \prime} p^{\prime \prime \prime}, h h^{\prime}}+\left\langle h^{\prime \prime} h^{\prime \prime \prime}\left|\widetilde{V}_{\mathrm{h}-\mathrm{h}}\right| h h^{\prime}\right\rangle d_{p p^{\prime}, h^{\prime \prime} h^{\prime \prime \prime}} .
\end{aligned}
$$

To simplify the calculation, we retain only those terms that survive in the limit of a boson theory: This implies the neglect of exchange effects and also of all contributions of the "ladder" type (which are invoked through the Jastrow correlation operator $F$ and are exactly accounted for in the Bose case). In the language of commonly used Feynman diagrams this amounts to keeping the proper polarization parts shown in Fig. 11 Clearly, the graphs no. 3 and 5-7 invoke vertex corrections, which are beyond the CRPA formalism.

We also assume a local pair excitation operator, i.e.

$$
d_{p p^{\prime} h h^{\prime}} a_{p}^{\dagger} a_{p^{\prime}}^{\dagger} a_{h^{\prime}} a_{h} \longrightarrow \sum_{q q^{\prime}} d\left(\mathbf{q}, \mathbf{q}^{\prime}\right)\left[\hat{\rho}_{\mathbf{q}} \hat{\rho}_{\mathbf{q}^{\prime}}-\hat{\rho}_{\mathbf{q}+\mathbf{q}^{\prime}}\right],
$$

where $\hat{\rho}_{\mathbf{q}}$ is the density operator, and further simplify the $c$ - $d$ coupling by replacing the amplitudes $c_{p h}(t)$ by their Fermi-sea averages. With these approximations the EOMs can be solved for $c_{p h}$ and $d_{p h, p^{\prime} h^{\prime}}$ which, in turn, yield the linear response wavefunction and thus the induced density $\delta \rho(\mathbf{r}, t)$.

Introducing

$$
\chi_{0}^{( \pm)}(q, \omega)= \pm \frac{1}{N} \sum_{h} \frac{n_{h} \bar{n}_{\mathbf{h}+\mathbf{q}}}{\hbar \omega \mp e_{h+q, h}+i 0^{+}},
$$

(where $\left.n_{k}=\theta\left(k_{\mathrm{F}}-k\right) \equiv 1-\bar{n}_{k}\right)$, the final result for the density-density response function can be expressed as follows:

$$
\chi^{\text {Pair }}(q, \omega)=\frac{\kappa(q, \omega)}{1-\kappa(q, \omega) \widetilde{V}_{\mathrm{p}-\mathrm{h}}(q)}
$$




\section{H. M. Böhm, E. Krotscheck, and M. Panholzer}

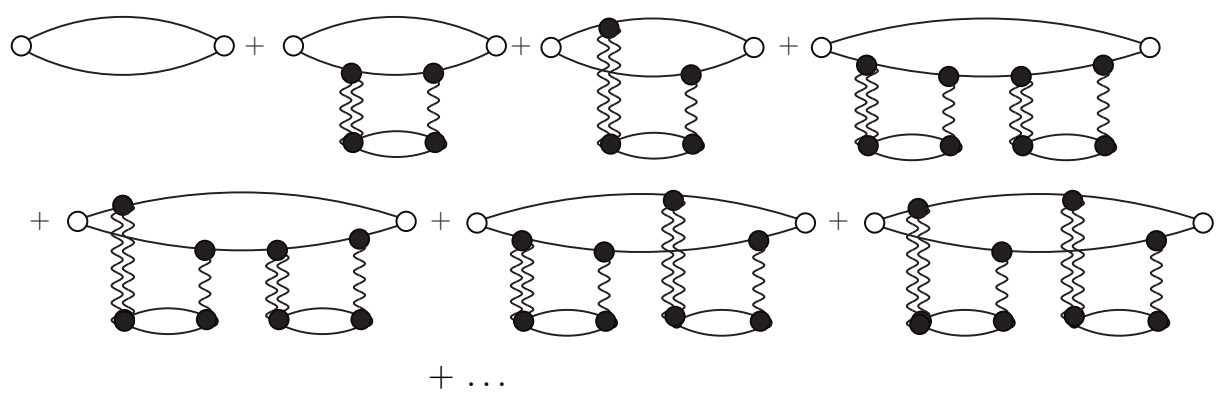

Fig. 1. Typical proper polarization Feynman diagrams taken into account in our approach. (The single and double wavy lines denote the bare and screened interactions, respectively. The lines with arrows denote free single particle propagators).

$$
\begin{gathered}
\kappa(q, \omega)=\frac{\chi_{0}^{(+)}}{1-\chi_{0}^{(+)} \mathcal{W}_{+}}+\frac{\chi_{0}^{(-)}}{1-\chi_{0}^{(-)} \mathcal{W}_{-}} \\
\mathcal{W}_{ \pm}=\mathcal{W}(q, \mp \omega)=\frac{1}{2} \sum_{\mathbf{q}^{\prime}} \frac{\overline{\mathcal{W}}_{3}\left(\mathbf{q}-\mathbf{q}^{\prime}, \mathbf{q}^{\prime}\right) \overline{\mathcal{W}}_{3}\left(\mathbf{q}-\mathbf{q}^{\prime}, \mathbf{q}^{\prime}\right)}{\mp \hbar \omega+\varepsilon\left(\mathbf{q}-\mathbf{q}^{\prime}\right)+\varepsilon\left(q^{\prime}\right)}
\end{gathered}
$$

Here $\overline{\mathcal{W}}_{3}$ is a three- "phonon" vertex, and $\varepsilon(q)=\hbar^{2} q^{2} /(2 m S(q))$ is the Feynman spectrum.

We conclude this section by noting that it is easily proved that $\chi(q, \omega)$ obeys the $\omega^{0}$ and $\omega^{1}$ sum rules, independent of the specifics of $\mathcal{W}(q, \omega)$. This means that $\widetilde{V}_{\mathrm{p}-\mathrm{h}}(q)$ is uniquely defined by the static structure function through the first two energy weighted sumrules.

\section{RESULTS}

In Fig. 2 we show the results for the dynamic structure factor $S(k, \omega)$ of ${ }^{3} \mathrm{He}$. Input to all our calculations was the static $S(k)$ obtained in Ref. 9]. The "correlated RPA" (= omitting pair excitations) is shown on the left; as mentioned in the introduction the collective mode is higher than found experimentally. The right picture gives the $\mathrm{CBF}$ result (= including pair fluctuations). Obviously, the dispersion is significantly improved and a weak pair-excitation background can be seen outside the patricle-hole continuum. As the calculated zero-sound mode is practically identical with the experimental one, a modification of the effective mass is unnecessary to obtain this result. 
Pair Excitations in Fermi Fluids
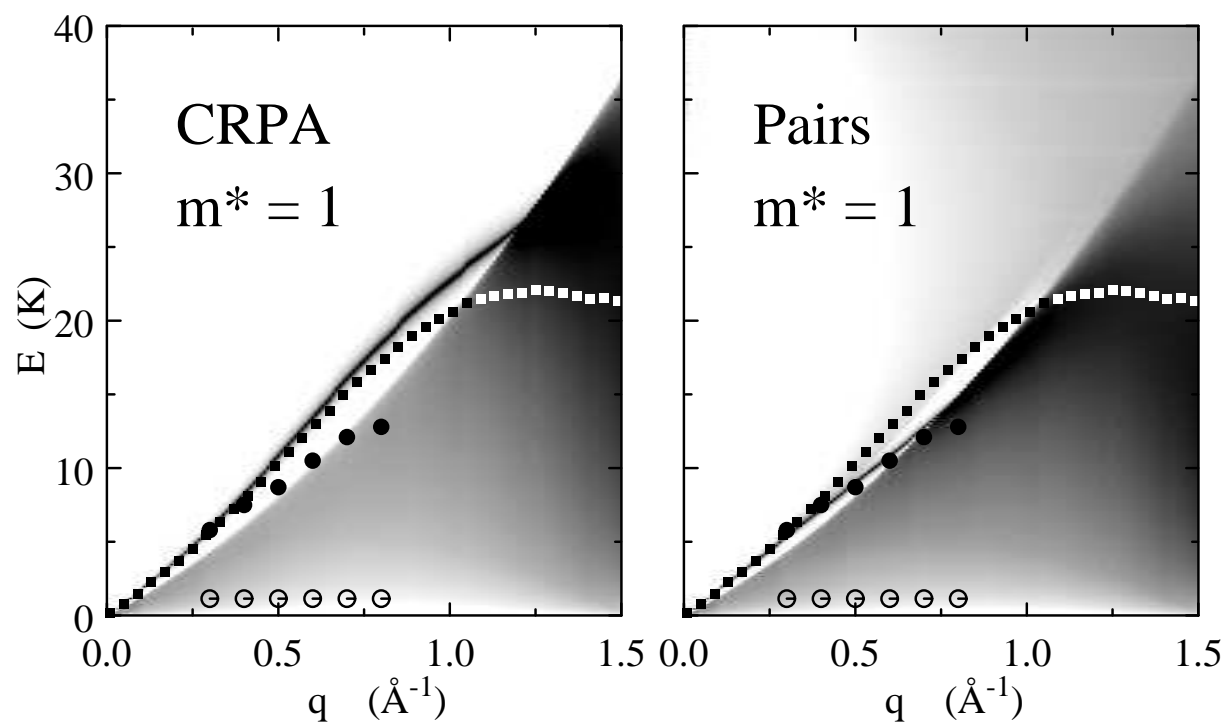

Fig. 2. Saturation density ${ }^{3} \mathrm{He}$ dynamic structure factor. Dark line: theoretical zero-sound mode. Filled [open] dots: experimental phonon [magnon] modes (Ref. ${ }^{4}$ ). Squares: RPA.

It appears interesting to investigate the effective mass of quasi-particle excitations in the system within this same formalism and to compare the result with the phenomenological $m^{*}$ used in Ref. 4. This can be done by studying the time dependent wave function corresponding to a definite single particle-hole amplitude, i.e. by omitting the sum over $p h$ (only) in the $c_{p h}$-contribution in Eq. 3. Then one can proceed in exactly the same way as above, leading to a consistent way of studying collective excitations and quasi-particle properties. Work in this direction is under investigation.

In summary, the introduction of dynamic multi-pair correlations, so successful in ${ }^{4} \mathrm{He}$, also proves necessary for obtaining the correct phonon dispersion in ${ }^{3} \mathrm{He}$. A promising extension is the inclusion of spin fluctuations, which holds the potential of understanding the large effective mass in ${ }^{3} \mathrm{He}$.

As a matter of course, the theory can also be applied to other strongly interacting Fermi fluids and to electrons.

\section{ACKNOWLEDGMENTS}

This work was supported by the FWF project P18134-N08. We thank H. Godfrin for providing the data ${ }^{7}$. 


\section{H. M. Böhm, E. Krotscheck, and M. Panholzer}

\section{REFERENCES}

1. R. P. Feynman and M. Cohen, Phys. Rev., 102, 1189-1204 (1956).

2. H. W. Jackson, Phys. Rev. A, 9, 964-975 (1974).

3. V. Apaja and M. Saarela, Phys. Rev. B, 57, 5358 (1998).

4. H. R. Glyde, B. Fåk, N. H. van Dijk, H. Godfrin, K. Guckelsberger, and R. Scherm, Phys. Rev. B, 61, 1421-1432 (2000).

5. N. Iwamoto and D. Pines, Phys. Rev. B, 29, 3924-3935 (1984)

6. K. S. Singwi and M. P. Tosi, Solid State Phys., 36, 177-266 (1981)

7. H. Godfrin, M. Meschke, and H. J. Lauter, (private communication).

8. E. Feenberg, Theory of Quantum Fluids, New York: Academic Press, 1969.

9. E. Krotscheck, J. Low Temp. Phys., 119, 103 (2000). 